\title{
Economic evaluation of genomic selection in small ruminants: a sheep meat breeding program
}

\author{
F. Shumbusho ${ }^{1,2 a}$, J. Raoul ${ }^{1}$, J. M. Astruc ${ }^{1}$, I. Palhiere ${ }^{2}$, S. Lemarié ${ }^{3}$, A. Fugeray-Scarbel ${ }^{3}$ and \\ J. M. Elsen ${ }^{\dagger}$ \\ ${ }^{1}$ Institut de I'Elevage, F-31321 Castanet-Tolosan, France; ${ }^{2}$ INRA, INPT ENSAT, INPT ENVT, UMR 1388 GenPhySE, F-31326 Castanet-Tolosan, France; ${ }^{3}$ INRA, UMR \\ GAEL, Univ. Grenoble Alpes, 38040 Grenoble Cedex 9, France
}

(Received 26 March 2015; Accepted 28 August 2015; First published online 8 October 2015)

\begin{abstract}
Recent genomic evaluation studies using real data and predicting genetic gain by modeling breeding programs have reported moderate expected benefits from the replacement of classic selection schemes by genomic selection (GS) in small ruminants. The objectives of this study were to compare the cost, monetary genetic gain and economic efficiency of classic selection and GS schemes in the meat sheep industry. Deterministic methods were used to model selection based on multi-trait indices from a sheep meat breeding program. Decisional variables related to male selection candidates and progeny testing were optimized to maximize the annual monetary genetic gain (AMGG), that is, a weighted sum of meat and maternal traits annual genetic gains. For GS, a reference population of 2000 individuals was assumed and genomic information was available for evaluation of male candidates only. In the classic selection scheme, males breeding values were estimated from own and offspring phenotypes. In GS, different scenarios were considered, differing by the information used to select males (genomic only, genomic + own performance, genomic + offspring phenotypes). The results showed that all GS scenarios were associated with higher total variable costs than classic selection (if the cost of genotyping was 123 euros/animal). In terms of AMGG and economic returns, GS scenarios were found to be superior to classic selection only if genomic information was combined with their own meat phenotypes (GS-Pheno) or with their progeny test information. The predicted economic efficiency, defined as returns (proportional to number of expressions of AMGG in the nucleus and commercial flocks) minus total variable costs, showed that the best GS scenario (GS-Pheno) was up to 15\% more efficient than classic selection. For all selection scenarios, optimization increased the overall $A M G G$, returns and economic efficiency. As a conclusion, our study shows that some forms of GS strategies are more advantageous than classic selection, provided that GS is already initiated (i.e. the initial reference population is available). Optimizing decisional variables of the classic selection scheme could be of greater benefit than including genomic information in optimized designs.
\end{abstract}

Keywords: breeding programs, genomic selection, economic analysis, sheep

\section{Implications}

Albeit to a much lesser degree than in dairy cattle, genomic selection may be technically efficient and economically profitable in sheep. Rather than implementing new selection schemes only based on rams' genomic evaluation of the breeding values, genomic and phenotypic information recorded for rams candidates (e.g. meat performances) should be merged. In the example studied in the present paper, about half of the improvement seen when moving from the current

aPresent address: Rwanda Agriculture Board (RAB) P. O. Box 5016 Kigali, Rwanda.

${ }^{\dagger}$ E-mail: Jean-Michel.Elsen@toulouse.inra.fr situation to the best genomic plan may be obtained by a better organization of classical breeding schemes.

\section{Introduction}

Genomic selection (GS) has become possible due to highperformance genotyping technology that allows individuals to be typed for thousands of single nucleotide polymorphisms (SNPs), genetic markers distributed over the whole genome. Assuming that all quantitative trait loci are in linkage disequilibrium with at least one genotyped SNP, the breeding values of selection candidates may be estimated directly from the analysis of such markers (Meuwissen et al., 2001). Genomic evaluation is already used routinely in many 
dairy cattle breeding programs and is expected to be implemented in the near future in other species. Recent studies in sheep and goats reported that the genomic evaluation of young males was more accurate than polygenic, pedigree-based evaluation (Daetwyler et al., 2012; Duchemin et al., 2012; Baloche et al., 2013; Carillier et al., 2013 and 2014).

Several studies have focused on the benefits of GS over conventional selection strategies in terms of genetic gain in various types of farm animal breeding programs and great economic gains have been reported in dairy cattle (Schaeffer, 2006; Konig et al., 2009). These expected benefits were mainly due to a reduction of the generation interval, an increase in the accuracy of the estimated breeding values for young bulls and bull dams and a reduction in costs for progeny testing (Schaeffer, 2006). The breeding structures and biological conditions inherent to sheep breeding programs differ from those used for dairy cattle in many aspects. In particular, (1) the generation interval is relatively short, (2) only few males are progeny tested, (3) joint use of artificial insemination (Al) and natural service and use of fresh semen only affect the progeny testing capacity, (4) progeny testing is less expensive than in cattle and (5) costs of genotyping is high when compared with the economic value of the selection candidate. The expected technical and economic benefits of GS could therefore be lower than in dairy cattle. A recent study (Shumbusho et al., 2013) showed that, nevertheless, benefits in terms of genetic gain may be associated with GS in small ruminant breeding programs. However, the authors did not demonstrate that, in sheep, breeding programs with higher genetic gains actually resulted in improved economic efficiency. Indeed, the economic benefits of a breeding program depend on the genetic gain, but also on many other parameters such as the extent and timing of expression of those genetic gains, the economic value of one unit of genetic gain and the costs of all inputs.

The objectives of this study were to compare the costs, monetary genetic gains and returns of classic and GS plans, taking the example of the Mouton lle de France sheep meat breeding program as organized in France. In order to compare the different selection strategies, costs and returns were determined at the industry level because the genetic superiority generated in nuclear farms is distributed across both nucleus and production farms.

\section{Material and methods}

The Mouton lle de France breeding scheme is targeted at improving meat and maternal traits. The structure and all population parameters of this breeding scheme have been described previously by Shumbusho et al. (2013). Briefly, the population is made of $\sim 14000$ ewes belonging to many breeders, of which $70 \%$ are candidates to become dams of sires. In total, around 40 proven (used as Al rams) and 300 natural service rams are available to service within the population (i.e. each male mates about 30 females). Each year, 300 young males enter the central testing station, where they are evaluated on their meat performances. About Test $=20$ of these males are then progeny tested (each tested ram is required to have at least 20 progeny records). Females are evaluated on their maternal performances, that is prolificacy measured by the number of live born lambs per lambing and milking ability measured by the 10 to 30 days of age lamb growth rate.

The differences in costs and economic efficiency between classic and GS schemes were assessed by implementing two approaches. We first consider the scheme found by Shumbusho et al. (2013) that maximizes genetic progress by an optimized choice of decision variable levels without taking into account economic cost and returns. As fully described in Shumbusho et al. (2013), decision variables are quantities, which may be chosen when designing the selection scheme (e.g. number of males selection candidates, selection rates, etc.). The objective here is to analyze the economic consequences of this first type of maximization. Second, for each type of selection scenario as defined in the following section, varying the level of decision variables, we search for the scheme that maximizes the genetic progress or the economic returns for a given level of investment.

\section{Selection scheme scenarios}

Four different selection scenarios were studied in respect to variable costs and economic efficiency. The scenarios have been described previously by Shumbusho et al. (2013) and are summarized hereafter.

Classic. This is a classical selection scheme, where estimates of breeding values (EBV) were based on phenotypes and pedigree information. After selection on the parents' average EBV, young males were selected based on their meat index. The best males are then progeny tested creating, by Al, a set of daughters in farms. These rams are evaluated on a global index combining meat and maternal traits. This scenario was used as a reference for comparative analysis with genomic scenarios.

GS. This is a pure GS scenario. Young male selection candidates were genotyped and best reproducers selected on their genomic breeding value (GBV) of meat and maternal traits at an early age. This scenario was modeled to quantify the effect of reducing the generation interval and use of genomic information.

GS-Pheno. In this scenario, young male selection candidates were genotyped and phenotyped for meat traits. Then, best reproducers were selected on an index combining genomic and individual phenotype information for meat records. The aim of this scenario was to assess the usefulness of the GS strategy for both meat and maternal traits when meat phenotypes are available from an early age. 
GS-PT. This scenario also used the GS scenario procedures as a pre-selection step. After progeny testing, elite rams were selected using a single index that combined the meat and maternal records of their progeny.

For all selection scenarios, females were first selected based on their parents' average EBV and, in a second step, on their phenotypes. Males were genotyped and their GBV estimated. Genomic information was available for male selection candidates ( $M s$ males) only.

\section{Response to selection}

The breeding scheme was assumed to be in a steady state, that is, it had been implemented for long enough to provide a constant annual genetic gain and the same cost each year. Selection response was predicted with deterministic methods based on selection index theory (Hazel, 1943), extending the model developed by Shumbusho et al. (2013) to include multiple traits in each index. The model predicts selection accuracy and genetic superiority of selection candidates, accounting for their age and genetic level.

In this study, two indices were constructed to represent two groups of traits: meat and maternal traits, which are the improvement targets for many meat sheep breeding programs in France (including the Mouton lle de France breeding program). The meat index $\left(I_{b}\right)$ was a combination of the average daily gain, back fat depth and conformation score traits, and the maternal index $\left(I_{m}\right)$ included prolificacy (Pr) and milk value. The genetic parameters and economic values of these indices traits are provided in Table 1 and details on the formulae used for the indices are described in Supplementary Material S1. The heritabilities and correlations are those reported by Bibé et al. (2002) and the economic values and standard deviations (SD) are those reported by Guerrier et al. (2010).

Using the physical units of the phenotypic and genetic variances of the traits, and their economic values, the genetic progress was expressed in monetary unit per unit change of the index (i.e. AMGG).

Genomic information was modeled as a trait with a heritability of 1.0 , which was genetically correlated to the corresponding selection index (Dekkers, 2007). This genetic

Table 1 Economic values (a), genetic standard deviation (GSD), heritabilities (bold on diagonal), genetic (above diagonal) and phenotypic (below diagonal) correlations of the traits included in selection indices

\begin{tabular}{llllll}
\hline \hline & \multicolumn{5}{c}{ Traits } \\
\cline { 2 - 7 } & Pr & MV & ADG & BFD & Confs \\
\hline Economic value (a) & 0.48 & 0.94 & 0.005 & 9.41 & 7.31 \\
Genetic standard deviation (GSD) & 14.0 & 0.9 & 20.0 & 0.63 & 0.23 \\
$\begin{array}{l}\text { Heritabilities and correlations } \\
\quad \text { Prolificacy (Pr; \%) }\end{array}$ & 0.09 & 0.0 & 0.0 & 0.0 & 0.0 \\
$\quad$ Milk value (MV; kg) & 0.0 & $\mathbf{0 . 2}$ & 0.0 & 0.0 & 0.0 \\
$\begin{array}{l}\text { Average daily gain (ADG; g/j) } \\
\text { Back fat depth (BFD; mm) }\end{array}$ & 0.0 & 0.0 & 0.18 & 0.01 & 0.0 \\
$\quad$ Conformation score (Confs) & 0.0 & 0.0 & -0.08 & $\mathbf{0 . 2 9}$ & 0.11 \\
\hline \hline
\end{tabular}

correlation between the genomic information and the index was equal to the accuracy of genomic prediction, $r_{\mathrm{GBV}}$, which depends on the reference population. The $r_{\mathrm{GBV}}$ was predicted using formulae of Daetwyler et al. (2008):

$$
r_{\mathrm{GBV}}=\sqrt{\operatorname{nref} \times h^{2} /\left(\mathrm{nref} \times h^{2}+M_{e}\right)}
$$

where $M_{e}=2 N_{e} L / \log \left(4 N_{e} L\right)$ is the number of effective genome segments (Goddard, 2009), which depends on the effective population size of the considered population $\left(N_{e}\right)$ and the genome length in Morgans $(L)$, nref is the number of animals forming the reference population and $h^{2}$ is the heritability of the trait. As in Shumbusho et al. (2013), the length of the genome was fixed to $L=27$, and a medium-sized reference population (nref $=2000$ ) of genotyped and phenotyped animals and an effective population size of $N_{e}=200$ were used to calculate $r_{\mathrm{GBV}}$.

\section{Evaluation of the variable cost of different selection strategies}

Following the model and selection scenarios described in the recent study by Shumbusho et al. (2013), we predicted the annual total variable cost $(C)$ of adopting the different selection strategies. Only costs that vary among the various selection scenarios were considered. Those costs correspond to, on the one hand, genomic information costs and, on the other hand, all other decisional variables characterizing the selection scheme. Formally, the total variable cost, per year, for any selection scenario was given as: $C=\sum_{i} C v_{i} X_{i}+$ cgeno, in which $c v_{i}$ is the unit cost of the $i^{\text {th }}$ decision variable, $X_{i}$ the value or level of the decision variable and cgeno the costs related to genotyping and extra statistical analysis (genomic scenarios only). In more details, cgeno was equal to $c$ geno $=\operatorname{Genoc} \times\left(M_{s}+0.2 \times\right.$ nref $)+$ statc were Genoc is the cost of genotyping one individual and statc the fixed costs of statistical analyses on genomic information. Genotyping costs were those spent on male selection candidates and on the renewal of the reference population (a 20\% renewal rate was supposed here). The question of to what extent and how often the reference population should be renewed to maintain or improve the prediction accuracy is still open to discussion. The consensus is that the reference population should be updated, at least to maintain GBV accuracy. On the basis of the assumption that this was an established breeding program, the costs of forming an initial reference population were not included in the variable costs.

Except for the costs of genomic information, other costs can be divided into four categories: (i) costs related to maintenance, recording and loss in slaughter of male selection candidates: $\quad c$ male $=M s \times\left(M s c_{p}(1-q M s)+M s c_{m}\right)$ where $M s c_{p}$ is a unit cost for loss in slaughter, $M s c_{m}$ the costs of maintenance and recording male selection candidates, and $q M s$ the proportion of males selected as reproducers or males kept for further evaluation. (ii) costs of keeping male reproducers: $\quad$ crepro $=n E$ Elite $\times$ elitec $+n N S \times N S c$, where elitec and NSC are the unit costs of maintaining elites and natural service rams, respectively, and $n E l i t e$ and $n N S$ the 
number of elite and NS rams used in a given scenario. (iii) costs of Al: $c A l=p A I \times p A l c$ where $p A l$ is the number of Al doses and $p A l c$ is the cost per dose. (iv) costs related to progeny testing: ctest $^{\prime}=$ Test $\times\left(\right.$ testc $_{b}+$ test $\left._{m}\right)$, where testc $_{b}$ and test $_{m}$ are the costs per ram of progeny testing for meat and maternal traits, respectively, and Test the number of male progeny tested per year.

Table 2 lists the decisional variables with their unit costs $\left(c v_{i}\right)$ as well as the cost categories affected by these variables and shows which selection scenarios they have an impact on. Optimal values of decision variables corresponding to the maximum genetic gain described in Shumbusho et al. (2013) are detailed in F. Shumbusho's PhD dissertation (2014). On the basis of these values, and using the above formulae and unit costs, variable costs associated with classic and GS strategies were estimated (Table 3).

\section{Economic returns and efficiency of different selection strategies}

Returns from genetic improvement depend on (i) the magnitude of genetic change (e.g. annual genetic gain expressed in physical or monetary units) and (ii) the extent of each individual's expression of genetic value on both nucleus and production farms. In the case of the Mouton lle de France breeding program where selection targets to improve meat and maternal traits, the genetic change is reflected by the number of culled lambs on nucleus (NAn) and production $(N A h)$ farms and breeding females on nucleus (NFn) and production (NFh) farms. The extra genetic value of a lamb attributable to selection is only expressed once in meat traits, but, for maternal traits, the extra genetic value of a female may be expressed more than once because dams are generally used for several years. For females, this multiple expression is quantified by the factor $\sum_{i=a_{1}}^{a_{1}+n}\left(\frac{1}{1+d}\right)^{i}$, where $a_{1}$ is the age (number of time units) at which females start expressing genetic superiority, $n$ the number of times dams are used as reproducers and $d$ a discounting rate. Table 4 gives population parameters related to the number of animals used to achieve genetic progress. (iii) The time lag between the creation and start of expression of genetic superiority. On nucleus farms, this time lag was approximated by the mean generation interval $(\bar{L})$. On production farms, the time lag was linked to the rate of replacement from the nucleus $(r h)$ and was approximated by $\overline{L h}=\frac{\bar{L}}{r h^{\prime}}$ that is, the asymptotic mean generation interval between a selected cohort in the nucleus and its first descendants born

Table 3 Optimal values of decision variables for the modeled scenarios, at different optimization levels

\begin{tabular}{lcccccr}
\hline \hline & & \multicolumn{5}{c}{ Decision variable $^{3}$} \\
\cline { 3 - 7 } Scenario $^{1}$ & Optimization level $^{2}$ & Ms & Test & pAI & $n$ nElite & $n N S$ \\
\hline \multirow{2}{*}{ Classic } & Current & 300 & 20 & 5000 & 30 & 300 \\
& Optirest & 310 & 30 & 5000 & 17 & 120 \\
& Optifull & 380 & 48 & 8200 & 10 & 50 \\
GS & Current & 300 & - & 5000 & - & 300 \\
& Optirest & 400 & - & 5000 & - & 52 \\
& Optifull & 600 & - & 5900 & - & 50 \\
GS-Pheno & Current & 300 & - & 5000 & - & 300 \\
& Optirest & 420 & - & 5000 & - & 68 \\
& Optifull & 460 & - & 6000 & - & 50 \\
GS-PT & Current & 300 & 20 & 5000 & 20 & 300 \\
& Optirest & 320 & 36 & 5000 & 10 & 85 \\
& Optifull & 420 & 46 & 7800 & 11 & 50 \\
\hline \hline
\end{tabular}

${ }^{1}$ Classic $=$ phenotypic selection and progeny testing with index selection; $\mathrm{GS}=$ pure genomic selection; GS-pheno = combined selection on genomic information and a meat phenotype; GS-PT-index = enomic selection and progeny testing with index selection.

${ }^{2}$ Current $=$ decision variables were those currently used in the breeding program except for genomic information; Optirest $=$ decisional variables were optimized but Al constrained to its current level of use in the breeding unit; Optifull = all decision variables were optimized.

${ }^{3}$ Defined in Table 2.

Table 2 Decision variables, related costs and impact of the different costs on selection scenarios

\begin{tabular}{|c|c|c|c|c|c|c|c|c|}
\hline \multirow[b]{2}{*}{ Decision variable } & \multirow[b]{2}{*}{ Symbol } & \multirow[b]{2}{*}{ Related costs } & \multirow[b]{2}{*}{ Symbol } & \multirow[b]{2}{*}{$\begin{array}{l}\text { Unit costs } \\
\qquad\left(c v_{i}\right)\end{array}$} & \multicolumn{4}{|c|}{ Scenario impacted ${ }^{1}$} \\
\hline & & & & & Classic & GS & $\begin{array}{l}\text { GS- } \\
\text { Pheno }\end{array}$ & $\begin{array}{l}\text { GS- } \\
\text { PT }\end{array}$ \\
\hline \multirow[t]{2}{*}{ Number of male selection candidates } & Ms & $\begin{array}{l}\text { Loss in slaughter value for male selection } \\
\text { candidates }\end{array}$ & $M s c_{p}$ & 30 & $\mathrm{x}$ & $\mathrm{x}$ & $x$ & $\mathrm{X}$ \\
\hline & & Records and maintenance of males in $\mathrm{SCl}$ & $M s c_{m}$ & 100 & $x$ & $x$ & $x$ & $x$ \\
\hline \multirow[t]{2}{*}{ Number of progeny tested males/year } & Test & Progeny testing on meat trait & testc $_{b}$ & 1800 & $\mathrm{x}$ & & & $x$ \\
\hline & & Progeny testing on maternal trait & testc $_{m}$ & 1000 & $x$ & & & $x$ \\
\hline Total number of elite males & nElite & Maintenance of Elite rams per year & elitec & 400 & $\mathrm{X}$ & $\mathrm{X}$ & $\mathrm{X}$ & $X$ \\
\hline Total number of natural service males & $n N S$ & Maintenance of Natural service rams per year & NSC & 300 & $\mathrm{X}$ & $\mathrm{X}$ & $X$ & $X$ \\
\hline \multirow{3}{*}{$\begin{array}{l}\text { Number of female artificially } \\
\text { inseminated }\end{array}$} & $p A l$ & Cost of one Al dose & pAlc & 10 & $\mathrm{x}$ & $\mathrm{X}$ & $\mathrm{x}$ & $\mathrm{x}$ \\
\hline & & Cost of a genotype & Genoc & 123 & & $x$ & $x$ & $x$ \\
\hline & & Cost of extra statistical analyses per year & Statc & 3000 & & $X$ & $X$ & $X$ \\
\hline
\end{tabular}

${ }^{1}$ Classic = phenotypic selection and progeny testing with index selection; GS = pure genomic selection; GS-Pheno = combined selection on genomic information and a meat phenotype; GS-PT-index = genomic selection and progeny testing with index selection. 
Table 4 Parameters related to the number of animals used to estimate the genetic gain and to calculate revenues for the selection strategies

\begin{tabular}{lcc}
\hline \hline Parameters & Symbol & Value \\
\hline Number of females on nucleus farms & $N F n$ & 14000 \\
Number of lambs born on nucleus farms & $N a n$ & 18200 \\
Number of females on commercial farms & $N F h$ & 200000 \\
Number of lambs born on commercial farms & $N a h$ & 220000 \\
Rate of dissemination of genetic gain to & $r_{h}$ & 0.5 \\
$\quad$ commercial population & $T$ & 30 \\
Investment period & $T$ & 0.05 \\
Discounting rate & $d$ \\
\hline \hline
\end{tabular}

on the production farms (a proportion $r h$ of these descendants are offspring, $r h(1-r h)$ grand offspring, etc.) This approximation is rather conservative because it neglects the erratic results observed over the first few years (Elsen and Mocquot, 1974; Hill, 1974). (iv) The time horizon $T$ that is, the moment when any prediction about the organization of the selection scheme looks too fragile (here 30 years).

The returns from selection on maternal traits $\left(R_{m}\right)$ and on meat traits $\left(R_{b}\right)$ are given as:

$$
\begin{aligned}
R_{m}= & A M G G_{m}\left\{\sum_{i=a_{1}}^{a_{1}+n}\left(\frac{1}{1+d}\right)^{i}\right\} \\
& \left\{N F n \sum_{t=\bar{L}}^{T}\left(\frac{1}{1+d}\right)^{t}+N F h \sum_{t=\overline{L h}}^{T}\left(\frac{1}{1+d}\right)^{t}\right\} \\
R_{b}= & A M G G_{b}\left\{N A n \sum_{t=\bar{L}}^{T}\left(\frac{1}{1+d}\right)^{t}+N A h \sum_{t=\overline{L h}}^{T}\left(\frac{1}{1+d}\right)^{t}\right\}
\end{aligned}
$$

which result in a total return $R=R_{m}+R_{b}$ and a contribution margin $C M=R-C$. Similarly to Shumbusho et al. (2013), the costs, returns and contribution margins were compared across scenarios in three different situations: (1) with the current values of the decision variables ('Current' situation), (2) with all the decision variables in the nucleus optimized except the proportion of Al ('Optirest' situation), and (3) with all decisions variables optimized ('Optifull' situation). Situation 3 allows a fair comparison between scenarios. Changes from situation 1 to 3 demonstrate the interest of optimization within scenario $v$. full reorganization of selection schemes adopting GS. Scenario 2 is an alternative to scenario 3 when avoiding changes in Al use, as the development of this technics is still limited by many factors, such as its cost and use of fresh semen in sheep. In Shumbusho et al. (2013), this procedure was applied using AMGG as the objective function, submitted to constraints between decision variables. In the present study, two approaches were followed: assessment of the economic efficiency of these technically optimized schemes (variable levels given in Table 3) and estimation of the maximum profitability (using either maximum $R$ or $C M$ ) at a given $C$ level. The second approach was justified by the observation
Table 5 Detailed variable costs in keuros for two different genotyping

\begin{tabular}{|c|c|c|c|c|c|c|c|c|}
\hline \multirow[b]{2}{*}{ Optimization level ${ }^{1}$} & \multirow[b]{2}{*}{ Costs $^{2}$} & \multirow[b]{2}{*}{ Classic $^{3}$} & \multicolumn{2}{|l|}{$\mathrm{GS}^{4}$} & \multicolumn{2}{|c|}{ GS-Pheno $^{5}$} & \multicolumn{2}{|c|}{ GS-PT } \\
\hline & & & 123 & 70 & 123 & 70 & 123 & 70 \\
\hline \multirow[t]{6}{*}{ Current } & $C$ & 246 & 271 & 234 & 271 & 234 & 336 & 298 \\
\hline & cgeno & & 89 & 52 & 89 & 52 & 89 & 52 \\
\hline & cmale & 34 & 34 & 34 & 34 & 34 & 34 & 34 \\
\hline & crepro & 106 & 98 & 98 & 98 & 98 & 106 & 106 \\
\hline & $c A l$ & 50 & 50 & 50 & 50 & 50 & 50 & 50 \\
\hline & ctest & 56 & & & & & 56 & 56 \\
\hline \multirow[t]{6}{*}{ Optirest } & $C$ & 221 & 223 & 181 & 233 & 190 & 264 & 226 \\
\hline & cgeno & & 101 & 59 & 103 & 60 & 91 & 53 \\
\hline & cmale & 41 & 51 & 51 & 53 & 53 & 39 & 39 \\
\hline & crepro & 48 & 21 & 21 & 26 & 26 & 32 & 32 \\
\hline & $c A l$ & 50 & 50 & 50 & 50 & 50 & 50 & 50 \\
\hline & ctest & 81 & & & & & 50 & 50 \\
\hline \multirow[t]{6}{*}{ Optifull } & $C$ & 243 & 283 & 230 & 249 & 204 & 337 & 294 \\
\hline & cgeno & & 126 & 73 & 108 & 63 & 103 & 60 \\
\hline & cmale & 47 & 77 & 77 & 58 & 58 & 53 & 53 \\
\hline & crepro & 24 & 21 & 21 & 22 & 22 & 24 & 24 \\
\hline & $C A I$ & 82 & 59 & 59 & 60 & 60 & 78 & 78 \\
\hline & ctest & 89 & & & & & 78 & 78 \\
\hline
\end{tabular}
costs (123 or 70 euros) for different selection schemes at different optimization levels

${ }^{1}$ Current $=$ decision variable levels as observed in Mouton lle de France; Optirest $=$ decision variables maximizing the genetic gain (all but the proportion of $\mathrm{Al}$ in the nucleus); Optifull = decision variables maximizing the genetic gain.

${ }^{2}$ cgeno $=$ costs related to genomic information; $c$ male $=$ costs related to male selection candidates; crepro $=$ costs of keeping male reproducers; $\mathrm{cAl}=$ costs of artificial insemination; ctest $=$ costs of progeny testing.

${ }^{3}$ Selection scheme without genomic information.

${ }^{4}$ Selection of rams on genomic information only.

${ }^{5}$ Selection of rams on genomic information and meat phenotypes.

${ }^{6}$ Selection of rams on genomic information and maternal phenotypes (after progeny testing).

that, when costs are not taken into account in the maximization program, the optimal scheme that are obtained leads to very different total variable cost. It is hence interesting to analyze how these results are affected when maximizing the AMGG at given level of variable costs.

At the time when this work was done, the cost of genotyping an individual with the OvineSNP50 BeadChip array was $\sim 123$ euros; therefore, the 123 euros costs was considered when estimating in the section dealing with optimized returns at given total variable costs. However, a lower cost of 70 euros was also considered when comparing scenario technically optimized.

\section{Results}

\section{Economic consequences of maximizing AMGG without cost constraint}

We first consider four selection scenarios where AMGG was maximized without taking into account the economic consequences (cost and returns). Table 5 gives the detailed variable costs for the two possible genotyping costs. Comparing the total variable cost of each scenario at any optimization level (i.e. Current, Optirest or Optifull) shows 
that all genomic scenarios are more expensive than classical selection if the genotyping cost is 123 euros, but less expensive than classical selection if genotyping only costs 70 euros (except for the GS-PT scenario). The GS-PT scenario is the most expensive because, in addition to costs common to all scenarios, it also includes the costs of both genotyping and progeny testing.

The AMGG and corresponding returns of the different selection strategies are given in Table 6. For all scenarios, genetic progress and returns were higher for the meat traits than for maternal traits. This can be explained in part by the fact that the variance and accuracy of the meat index were higher compared with the maternal index. From the contribution margins (CM) criteria, the highest economic benefits were observed with the GS-Pheno scenario. All genomic scenarios were superior to classic selection (Classic), except for the GS scenario at the 'Optifull' level of optimization. However, apart from the GS-Pheno scenario, the benefits remain limited. The impact of an increase of the quantity of Al doses depends on the structure of the selection scheme. More precisely, increasing the quantity of Al doses is beneficial (higher $\mathrm{CM})$ only for selection scheme with progeny testing $(10.6 \%$ and $8.8 \%$ higher CM for Classic and GS-PT, respectively). For all scenarios, optimization increased overall AMGG, returns and CM. For the classic selection scenario, optimizing the use of decisional variables was more beneficial than including genomic information in an optimized classic design.

\section{Optimized returns at given total variable costs}

The optimized overall AMGGs are plotted in Figure 1 and the corresponding returns in Figure 2. Increasing the maximum annual variable costs increased the overall AMGG and returns of all selection scenarios. Trends were globally similar, but it is interesting to note that GS-PT produced a higher AMGG but lower expected returns than GS-Pheno when annual variable costs were over 260 keuros. The plateau, when returns remain constant as costs continue to increase, was reached at $\sim 180$ keuros for the GS scenario, 240 keuros for the Classic and GS-Pheno scenarios, and 300 keuros for the GS-PT scenario. For the GS scenario, once the level of annual variable costs is sufficient to cover all the genotyping needs ( 180 keuros), its AMGG reaches a maximum level and no extra returns can be obtained. For the GSPheno scenario, the plateau is reached at a higher level of costs because increasing the number of phenotyped young rams continues to result in extra genetic gains in meat traits. For both classic and GS-PT scenarios, a better efficiency of progeny testing when more resources are available explains the shift of the plateau towards higher annual variable costs.

Optimized returns were, at almost all investment levels, highest for the GS-Pheno and GS-PT scenarios. The results shown in Figure 3 demonstrate that in terms of economic

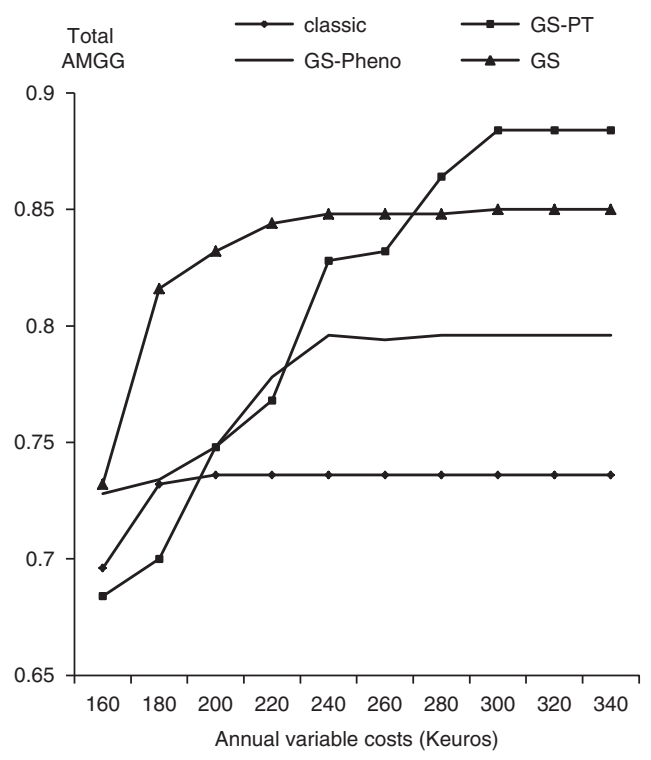

Figure 1 Optimized total Annual Monetary Genetic Gain (AMGG on meat + AMGG on maternal traits) at given total variable costs for different selection scenarios of the breeding program.

Table 6 Annual monetary genetic gain for meat $\left(A M G G_{b}\right)$ and maternal $\left(\mathrm{AMGG}_{\mathrm{m}}\right)$ indices, returns $\left(\mathrm{R}_{\mathrm{b}}, \mathrm{R}_{\mathrm{m}}, \mathrm{R}=\mathrm{R}_{\mathrm{b}}+\mathrm{R}_{\mathrm{m}}\right)$ and contribution margins (CM) in Meuro, for different selection strategies

\begin{tabular}{lccccccc}
\hline \hline Scenario $^{1}$ & Optimization level $^{2}$ & $A M G G_{b}$ & $A M G G_{m}$ & $R_{b}$ & $R_{m}$ & $R$ \\
\hline Classic & Current & 0.440 & 0.156 & 1.029 & 0.240 & 1.269 & 1.022 \\
& Optirest & 0.509 & 0.178 & 1.190 & 0.275 & 1.464 & 1.243 \\
GS & Optifull & 0.556 & 0.216 & 1.301 & 0.334 & 1.634 & 1.390 \\
& Current & 0.438 & 0.205 & 1.025 & 0.315 & 1.340 & 1.068 \\
& Optirest & 0.515 & 0.210 & 1.204 & 0.324 & 1.528 & 1.304 \\
GS-Pheno & Optifull & 0.545 & 0.194 & 1.276 & 0.299 & 1.575 & 1.291 \\
& Current & 0.533 & 0.194 & 1.247 & 0.298 & 1.546 & 1.274 \\
& Optirest & 0.630 & 0.198 & 1.473 & 0.305 & 1.777 & 1.544 \\
GS-PT & Optifull & 0.639 & 0.191 & 1.495 & 0.294 & 1.789 & 1.539 \\
& Current & 0.444 & 0.216 & 1.039 & 0.333 & 1.372 & 1.036 \\
& Optirest & 0.510 & 0.281 & 1.192 & 0.433 & 1.625 & 1.360 \\
& Optifull & 0.590 & 0.283 & 1.380 & 0.436 & 1.816 \\
\hline \hline
\end{tabular}

${ }^{1}$ Classic: phenotypic selection and progeny testing with index selection; GS = pure genomic selection; GS-Pheno = combined selection on genomic information and a meat phenotype; GS-PT-index = genomic selection and progeny testing with index selection.

${ }^{2}$ Current $=$ decision variables were those currently used in the breeding program except for genomic information; Optirest $=$ decisional variables were optimized but $\mathrm{Al}$ constrained to its current level of use in the breeding unit; Optifull = all decision variables were optimized. 


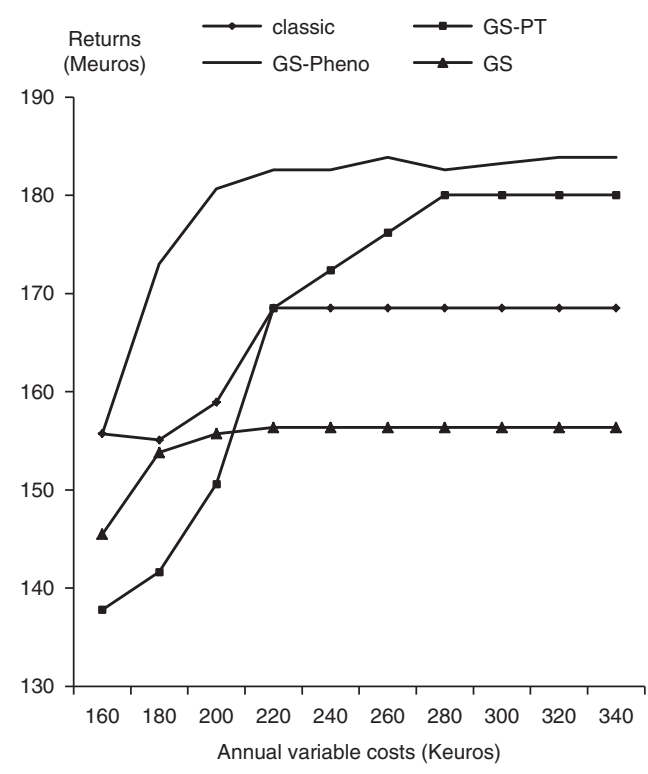

Figure 2 Total revenues of different selection scenarios of the breeding program at different levels of total variable costs.

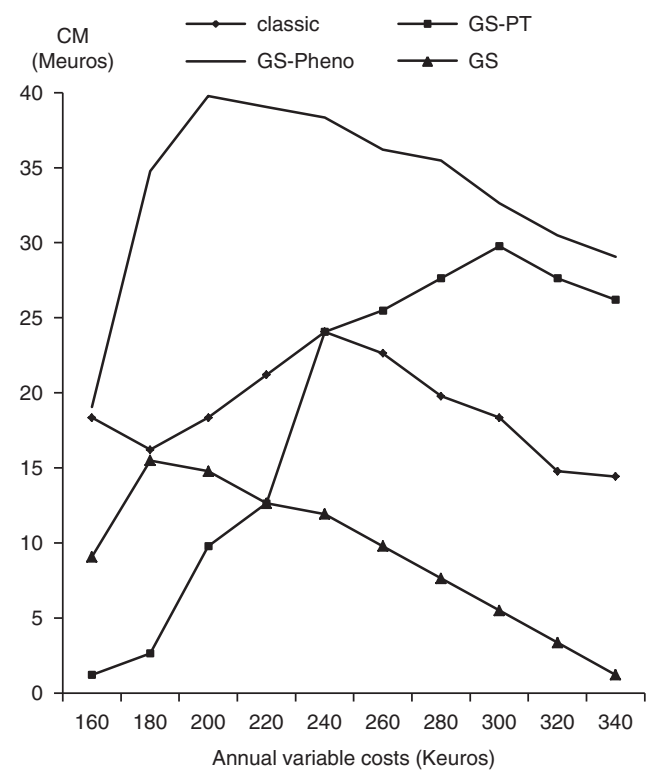

Figure 3 Contribution margins (CM) at different levels of total variable costs for different selection scenarios of the breeding program.

efficiency (contribution margin), the GS-Pheno scenario was the most efficient at all levels of total variable costs studied. All selection scenarios had an optimum cost level, with a contribution margin peak. This economically relevant efficiency peak was at lower cost levels for scenarios without progeny testing (180 and 200 keuros, respectively, for the GS and GSPheno scenarios) than for progeny testing scenarios (240 and 300 keuros, respectively, for the Classic and GS-PT scenarios).

\section{Discussion}

In this study, we modeled and optimized classic and GS in sheep with the example of the Mouton lle de France breeding program. Three GS scenarios were compared with the classic scenario: GS (males are only selected on GEBV), GS-Pheno (males are selected on an index combining meat phenotypes and GEBV), and GS-PT (males are first selected on GEBV, then on the results of a progeny test).

\section{Modeling options}

Assuming both classic and GS schemes were in routine use, comparisons were based on the variable costs incurred by each selection strategy and the economic returns resulting from genetic change. The dissemination of genetic progress in nucleus and commercial flocks was approximated using a discounting technique. The dynamics of the gene diffusion, in particular in multi-tier populations, is known to be not linear (Elsen and Mocquot, 1974; Hill, 1974) and could be modeled appropriately. Further investigations using such a model will help to balance the costs related to one cycle of selection and the returns resulting from the extra value of the individuals selected during this cycle, expressed by generations of progeny in the nucleus and in commercial farms. The most interesting feature of this dynamic modeling will be the possibility of describing transition phases, in our case the transition from classic to genomic schemes.

To be consistent with the Shumbusho et al. (2013) paper, the accuracy $r_{\mathrm{GBV}}$ of GS was predicted (1) using the Daetwyler et al. (2008) formulae, and (2) assuming the number of effective genomic segments $M_{e}$ follows the Goddard (2009) equation. The recent publication of Brard and Ricard (2015), based on a meta-analysis of 13 publications on GS, mainly in dairy cattle, showed how variables are the predictions of GS precision, and in particular strongly depend on the $r_{\mathrm{GBV}}$ and $M_{e}$ formulae chosen. They concluded that 'no rules can be applied to predict the reliability of GS using [compared] formulae'. From their simulations, the combination chosen here for $r_{\mathrm{GBV}}$ (Daetwyler et al., 2008) and $M_{e}$ (Goddard, 2009) predicts high accuracy in the situations they explored. However, with the parameters chosen in the present work $\left(L=27\right.$, nref $=2000$ and $N_{e}=$ $200)$, this accuracy is low, with an upper bound of 0.65 (corresponding to $h^{2}=1$ ). Moreover, with the genetic parameters supposed here (Table 1), and using Supplementary Material $\mathrm{S1}$, heritabilities of meat and maternal traits were 0.19 and 0.16 , and GEBV accuracies limited to 0.26 and 0.23 . These very low values are explained by the organization of the reference population, genotyped individuals being phenotyped on their own performances only.

Even if the size of the reference population $(n r e f=2000$ ) may appear large relatively to the recorded population size (14 000 females), it was needed to provide sufficient accuracy to GS. This reference population may be rapidly obtained for maternal traits, but needs some time for the meat traits, since only 300 to 600 new males are phenotyped each year. It was supposed that meat traits were measured on the replacement female lambs as well, allowing a faster creation of the reference population. This simplifying hypothesis is consistent with the $20 \%$ annual renewal, which 
corresponds to the female observed replacement rate in meat sheep population.

Other options should be considered for the creation and renewal of the reference population: 300 to 600 young males are performance tested each year, 20 to 48 rams are progeny tested using $\mathrm{Al}$ and 50 to 300 natural service rams get EBV from their daughters' performances. These opportunities would improve the quality of the reference population and the accuracy of GS. Returns on investment could be appreciated following the principle presented here.

As there are many uncertainties about the accuracy of GS, and to overcome all the simplifications done in the present paper, considering this accuracy as a parameter and searching for a threshold above which GS is profitable would be an other option we are presently working on.

\section{Costs}

Genomic costs included the cost of genotyping male selection candidates and a 20\% renewal of the reference population, as well as the cost of extra statistical analyses. Genomic scenarios were more expensive than the classic scenario. When scenarios were compared with the current level of decisional variables, costs of up to 89 keuros were found for obtaining genomic information (32.7\% of the total variable cost of the GS and GS-Pheno scenarios), whereas the cost of progeny testing was 56 keuros $(22.6 \%$ of the total variable cost of the Class-PT scenario). This means that implementing GS (even without progeny testing) in meat sheep breeding schemes similar to the Mouton lle de France program, will involve extra costs. A similar result was obtained by Banks and Van der Werf (2009) who concluded that 'implementing Genomic Selection in sheep should stimulate evolution of the industry structure towards a more clearly defined nucleus:multiplier:commercial base model'. This is quite different from what was predicted in dairy cattle breeding programs. Indeed, Schaeffer (2006) compared the cost of genotyping male selection candidates and dams of sires with the cost of progeny testing in dairy cattle breeding programs and predicted a $92 \%$ decrease in the cost of proving bulls (the author considered the cost of genotyping an individual at \$500). However, compared with our results, much higher extra costs were predicted in pig breeding programs. In this respect, Tribout et al. (2013) showed that in French pig breeding schemes, 'genomic selection would not result in organizational changes to the schemes that could generate sufficient savings to compensate for the cost of genotyping'.

If the cost of genotyping was of only 70 euros/animal, both our GS and GS-Pheno scenarios would be cheaper than classic selection. Such a reduction in the cost of genotyping is not necessarily unrealistic and could be envisaged in two different manners. First, with the constant developments in sequencing and genotyping technologies and the progressive decrease in genotyping costs, it is reasonable to expect that costs of genotyping will continue to decrease. Second, the costs related to genotyping could be reduced if selection candidates were genotyped on low density (thus low cost) chips and imputation techniques used. Imputation is already being used for genomic evaluation in dairy cattle and excellent results have been reported (Dassonneville et al., 2011; Huang et al., 2012).

Our modeling assumed the breeding schemes were in a steady state, and the cost of genotyping the initial reference population was not considered here. For a reference population such as ours of 2000 individuals, this cost amounts to 246 keuros and could be distributed over an investment period of 30 years (i.e. 8200 euros/year without discounting). This would slightly increase the cost of genomic scenarios, but would not change scenario rankings, and the predicted contribution margin comparisons would be only marginally affected. More generally, considering an investment period of 30 years may look optimistic in comparison with the speed of technological evolution. However, even if new technologies emerge in the future, we expect that the gain provided by GS will not be diminished but amplified.

\section{Economic returns and efficiency}

In general, GS-Pheno and GS-PT scenarios gave the highest AMGG and associated returns for two main reasons. In the GS-Pheno scenario, this was due to a short generation interval and sustained high accuracy of the index. Unlike the GS scenario where the accuracy depended only on genomic information, the GS-Pheno scenario combined both genomic information and a meat phenotype. This meant that high accuracy was achieved for the meat index and thus a highly accurate genetic trend was obtained. The superiority of the GS-PT scenario was due to genomic pre-selection for progeny-tested males. At a low investment level, progeny testing scenarios showed lower returns. Such scenarios are therefore of very limited interest if the progeny sample sizes are not large enough to correctly estimate the sires' breeding values. The difference in cost at which the maximum contribution margin is achieved between the Classic and GS-PT scenarios (300 v. 240 keuros) results from the need to invest both in genomic and progeny test information in the latter scenario. The fact that at high cost levels (over 280 keuros), the GS-PT scenario was superior to the GS-Pheno scenario with respect to the AMGG (Figure 1), but inferior in terms of economic return and CM (Figures 2 and 3), may be due to the longer generation interval in the GS-PT scenario. On average, the creation of genetic superiority (in breeding units) takes longer with the GS-PT scenario than with the GS-Pheno scenario and this affects the overall result when measured over the same investment period.

The GS-Pheno scenario was the most profitable, with maximum contribution margins increased by $\sim 15 \%$ compared with the classic selection scheme. Indeed, in addition to providing higher returns as a result of a higher annual genetic gain, the GS-Pheno scenario was relatively less expensive because, as for the GS scenario, the costs of progeny testing were saved. For the GS scenario, the costs of phenotyping male selection candidates at the central testing station were maintained in order to renew the reference population for the following generations. This affected the 
economic efficiency of the scenario because this meat phenotype was not used to improve selection accuracy. Obviously, if a phenotype can be recorded before the reproduction age, it should be combined with genomic information to improve selection accuracy and the predicted genetic gain. Although the GS-PT scenario gave high returns, its superiority in terms of contribution margins was lower because it incurred higher costs than other scenarios. Non-progeny testing scenarios reached their highest returns at relatively low costs and thus were most efficient at low investment levels.

In conclusion, this study shows that the implementation of some forms of genomic evaluation in small ruminant breeding programs can be more profitable than classic selection. However, these economic gains are relatively lower than the technical gains (annual genetic gain) predicted by Shumbusho et al. (2013) in sheep and goat breeding programs. Our results also confirm the need to optimize the use of decisional variables. Our comparisons were performed at the industry level without differentiating between revenues from nucleus or production farms. It would be interesting to further analyze in detail specific costs and benefits of such strategies for the different stakeholders of the sheep industry (i.e. breeding organizations, breeders and farmers).

\section{Acknowlegments}

This study is part of the CASDAR GENOVICAP project funded by the French Ministry of Agriculture. Support was also provided by the INRA Metaprogram Selgen. The authors gratefully acknowledge the funding.

\section{Supplementary material}

To view supplementary material for this article, please visit http://dx.doi.org/10.1017/S1751731115002049

\section{References}

Baloche G, Legarra A, Sallé G, Larroque H, Astruc J-M, Robert-Granie C and Barillet $F$ 2013. Assessment of the accuracy of genomic prediction for French Lacaune dairy sheep. Journal of Dairy Science 97, 1107-1116.

Banks RG and van der Werf JHJ 2009. Economic evaluation of whole genome selection using meat sheep as a case study. Proceedings of the 18th Conference of the Association for the Advancement of Animal Breeding and Genetics, 28 September-1 October 2009, Barossa Valley, South Australia, pp. 430-435.

Bibé B, Brunel J-C, Bourdillon Y, Loradoux D, Gordy MH, Weisbecker J-L and Bouix J 2002. Genetic parameters of growth and carcass quality of lambs at the French progeny-test station berrytest. Proceedings of the 7th World Congress of Genetics Applied to Livestock Production, 19-23 August 2002, Montpellier, France, pp.335-338.
Brard S and Ricard A 2015. Is the use of formulae a reliable way to predict the accuracy of genomic selection? Journal of Animal Breeding and Genetics 132, 207-217.

Carillier C, Larroque H, Palhiere I, Clement V, Rupp R and Robert-Granie C 2013. A first step toward genomic selection in the multi-breed French dairy goat population. Journal of Dairy Science 96, 7294-7305.

Carillier C, Larroque $\mathrm{H}$ and Robert-Granié C 2014. Comparison of joint versus purebred genomic evaluation in the French multi-breed dairy goat population. Genetic Selection Evolution 46, 67. doi:10.1186/s12711-014-0067-3.

Daetwyler H, Swan A, van der Werf J and Hayes BJ 2012. Accuracy of pedigree and genomic predictions of carcass and novel meat quality traits in multi-breed sheep data assessed by cross-validation. Genetics Selection Evolution 44, 33. doi:10.1186/1297-9686-44-33.

Daetwyler HD, Villanueva B and Woolliams JA 2008. Accuracy of predicting the genetic risk of disease using a genome-wide approach. PLoS One 3, e3395. doi:10.1371/journal.pone.0003395.

Dassonneville R, Brøndum RF, Druet T, Fritz S, Guillaume F, Guldbrandtsen B, Lund $M$, Ducrocq $V$ and Su G 2011. Effect of imputing markers from a low-density chip on the reliability of genomic breeding values in Holstein populations. Journal of Dairy Science 94, 3679-3686.

Dekkers JC 2007. Prediction of response to marker-assisted and genomic selection using selection index theory. Journal of Animal Breeding and Genetics 124, 331-341.

Duchemin SI, Colombani C, Legarra A, Baloche G, Larroque H, Astruc J-M, Barillet F, Robert-Granie C and Manfredi E 2012. Genomic selection in the French Lacaune dairy sheep breed. Journal of Dairy Science 95, 2723-2733.

Elsen J-M and Mocquot J-C 1974. Méthode de prévision de l'évolution du niveau génétique d'une population soumise à une opération de sélection et dont les générations se chevauchent. Bulletin Technique du Département de Génétique animale. INRA 17, 30-54.

Goddard ME 2009. Genomic selection: prediction of accuracy and maximisation of long term response. Genetica 136, 245-257.

Guerrier J, Praud J-P, Poivey J-P, Batut H, Grenet G and Bouix J 2010. Définition économique des objectifs de sélection en ovins allaitants. Application à la race Blanche du Massif Central. Proceedings of the 17th Rencontres Recherches Ruminants 17, pp. 443-446.

Hazel LN 1943. The genetic basis for constructing selection indexes. Genetics 28, 476-90.

Hill WG 1974. Prediction and evaluation of response to selection with overlapping generations. Animal Science 18, 117-139.

Huang Y, Hickey J, Cleveland M and Maltecca C 2012. Assessment of alternative genotyping strategies to maximize imputation accuracy at minimal cost. Genetics Selection Evolution 44, 25. doi:10.1186/1297-9686-44-25.

Konig S, Simianer $\mathrm{H}$ and Willam A 2009. Economic evaluation of genomic breeding programs. Journal of Dairy Science 92, 382-391.

Meuwissen THE, Hayes BJ and Goddard ME 2001. Prediction of total genetic value using genome-wide dense marker maps. Genetics 157, 1819-1829.

Schaeffer LR 2006. Strategy for applying genome-wide selection in dairy cattle. Journal of Animal Breeding and Genetics 123, 218-223.

Shumbusho F 2014. Designing, technical evaluation and profitability estimation of breeding strategies based on molecular information for small ruminant species. Thesis PhD, INP Toulouse University, Toulouse, France.

Shumbusho F, Raoul J, Astruc J-M, Palhiere I and Elsen J-M 2013. Potential benefits of genomic selection on genetic gain of small ruminant breeding programs. Journal of Animal Science 91, 3644-3657.

Tribout T, Larzul C and Phocas F 2013. Economic aspects of implementing genomic evaluations in a pig sire line breeding scheme. Genetics Selection Evolution 45, 40. doi:10.1186/1297-9686-45-40. 